\title{
Design and Analysis of Bayesian Model Predictive Controller
}

\author{
Yijian Liu ${ }^{1}$, Weixing Qian ${ }^{1} \&$ Liming $\mathrm{Di}^{1}$ \\ ${ }^{1}$ School of Electrical and Automation Engineering, Nanjing Normal University, Nanjing, China \\ Correspondence: Yijian Liu, School of Electrical and Automation Engineering, Nanjing Normal University, \\ Nanjing, China. Tel: 86-025-8548-1197. E-mail: 63055@njnu.edu.cn
}

Received: June 9, $2014 \quad$ Accepted: June 16, $2014 \quad$ Online Published: July 5, 2014
doi:10.5539/cis.v7n3p58
URL: http://dx.doi.org/10.5539/cis.v7n3p58

The research is supported by the the Jiangsu Government Scholarship for Overseas Studies (2013), the National Natural Science Foundation of China under Grant 61304227 and 61273114,the Innovation Program of Shanghai Municipal Education Commission under Grant 14ZZ087, the Pujiang Talent Plan of Shanghai City, China under Grant 14PJ1403800 and the Natural Science Foundation of Jiangsu Province of China under Grant BK20131403.

\begin{abstract}
In this article, a novel predictive controller based on a Bayesian inferring nonlinear model (BMPC) is presented and analyzed. In the construction of the BMPC, the Bayesian inferring model is selected as the predictive model with the characteristics of on-line tracing ability to the actual controlled object. The nonlinear programming method called the steepest gradient is set as the receding horizon optimization algorithm of the BMPC. The on-line controller output is obtained using this method. The convergence analysis of the proposed BMPC is given and the examples (nonminimum phase and nonlinear objects) are selected to validate the performance of the BMPC. The simulation results show that with the help of the presented BMPC algorithm, the closed loop control system demonstrates the abilities of anti-disturbance and robustness.
\end{abstract}

Keywords: Bayesian predictive control, nonlinear system, modeling, receding horizon optimization

\section{Introduction}

The model predictive control (MPC) method, derived from the practice industrial application, has been widely used in many industrial fields such as petroleum industry, chemical industry and pharmaceutical manufacturing industry (Armando, 2013; Anders, 2013; Ali, 2012). The reason for the success of the MPC is that the MPC method owns three characteristics, namely, predictive model, feedback correction and rolling optimization. Among them, the predictive model is not limited to any special model framework. And any model, which can provide the ability to predict the system output, all can be used as the predictive model. So the extensity of the predictive model contributes to the research prosperity of the MPC (Aswani, 2013; Rodriguez, 2013; Giselsson, 2013).

The predictive model plays an essential role to the predictive accuracy of the MPC. In the past decades, the theory of linear model predictive control based on linear predictive model has already been fully researched. But in industrial control, the majority of controlled objects possess many kinds of nonlinear characteristic. So the research of nonlinear model predictive control is very meaningful and practical. Until now, there is no uniform model describing method for all kinds of nonlinear systems in nonlinear model predictive research. Commonly, three design ideas are presented till now. The first idea is to make the nonlinear model linearization and then to solve the problem based on the developed linear predictive model control method (Christofides, 2013; Lincoln, 2013; Tung, 2013). The second idea is to obtain the nonlinear system model using identification method and converted the controller design problem into nonlinear programming issue. And then the control variable is optimized with the aid of some receding horizon methods. The nonlinear first-principles modeling method is also been used (Mesbah, 2010).

In the second research idea of nonlinear model predictive control, the fuzzy model and neural network (Xiangjie, 2013; Hazil, 2014; Karim, 2013) are two kinds of frequently used nonlinear models. But the designs of membership functions and fuzzy rules all have a great influence on the predictive accuracy. In the design of neural network model, the number of hidden layers and nodes and the dynamic or static neural structure are all 
determined by designer experience. All in all, the fuzzy model and neural network are all preset and use the obtained data sample to train the model structure parameters. From another perspective, the nonlinear characteristics are exhibited through the input and output data. So we should focus on the data directly in the design of nonlinear predictive model.

The modeling method based on statistical theory is a kind of direct modeling solution, which utilizes the sample data to obtain the relationship between the input and output of the research system. The Bayesian Gauss neural network based on Bayesian inferring method directly utilizes the input and output data to identify the system nonlinear relationship. The former research (Liu, 2010) shows that the Bayesian Gauss neural network demonstrates the on-line modeling and tracing abilities.

The novelty of this article is to present a kind of Bayesian model predictive controller (BMPC). The Bayesian Gauss neural network is selected as the predictive nonlinear model. And the design procedures of the BMPC and performance analysis are also given in details. This article is organized as follows. Section 2 describes the structure of the proposed BMPC and detail design procedures of the BMPC. Section 3 described the experiments of the BMPC on the control of the nonminimum phase and nonlinear objects. The results and discussion are also given at section 4 and 5 . The conclusion is then presented at section 6 finally.

\section{Design of Bayesian Model Predictive Controller}

\subsection{Structure of the BMPC System}

The abstract structure of the Bayesian model predictive control system is shown in figure 1.

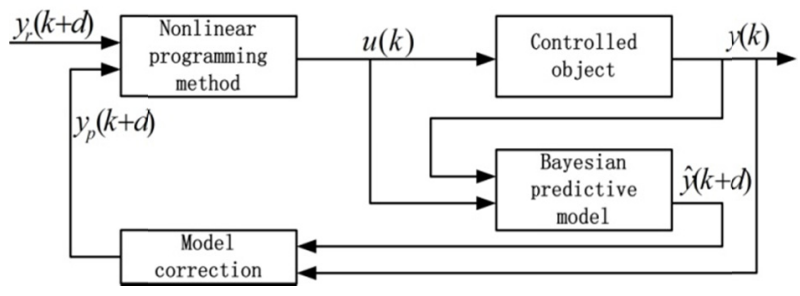

Figure 1. Structure of Bayesian model predictive control system

The components of the BMPC system include four blocks of the controlled object, Bayesian Gauss predictive model, model correction and nonlinear programming. $u$ denotes the output of the controller at the moment of $k ; y_{r}(k+d)$ and $\hat{y}(k+d)$ are the $d^{\text {th }}$ step reference input and system predictive output respectively. $y_{p}(k+d)$ is the $d^{\text {th }}$ step corrected system output.

Because there are many disturbances in actual industrial process control, the established system model has much uncertainty inevitably. In the design of predictive model control system, the uncertainty should be corrected. The correction method adopts the following algorithm.

$$
y_{r}(k+d)=\hat{y}(k+d)+e(k)
$$

Where $e(k)=y(k)-\hat{y}(k)$ denotes the predictive error at the moment of $k$. Using the correction method, the uncertainty is compensated to some extent and this mechanism also enables the model predictive control to become a feedback control system.

\subsection{Bayesian Gauss Predictive Model}

The Bayesian Gauss inferring model is adopted as the nonlinear predictive model in this paper. The structure of the Bayesian Gauss inferring model (Liu, 2010)is shown in figure 2. The input and output of nonlinear system are denoted as $(u(i), y(i)), i=1,2 \ldots, N$ and $N$ is the maximum sample number.

At the $k^{\text {th }}$ sample moment, $X_{k}=[u(k-1), u(k-2), \ldots, u(k-m), y(k-1), y(k-2), \ldots, y(k-n)]^{\Gamma}$ is the input vector of the Bayesian inferring model. $m$ and $n$ are the related coefficients about the input and output variables respectively. Based on the bayesian formula and gaussian assumption, the above bayesian inferring model can be realized through the following equations (Liu, 2010).

$$
\hat{y}(k)=\sigma(k)^{2} \sum_{i=1}^{N} \sigma_{i}^{-2} y(i)
$$




$$
\begin{aligned}
& \sigma^{-2}(k)=\sum_{i=1}^{N} \sigma_{i}^{-2} \\
& \sigma_{i}^{2}=\sigma_{0}^{2} e^{\left(X_{k}-X_{i}\right)^{T} D\left(X_{k}-X_{i}\right)} \\
& D=\operatorname{diag}\left[d_{11}^{-2}, d_{22}^{-2}, \ldots, d_{m m}^{-2}\right]
\end{aligned}
$$

Where, in above equation, $X_{i}$ denotes the history Bayesian input vector similar to $X_{k} . \sigma_{i}$ is variance variable according to the sample vector $X_{i}$. The $D$ is called threshold matrix. $D$ is diagonal matrix $(m m=m+n+1)$ with the same dimension as the number of the elements of input vector $X_{k}$.

The training algorithms of the Bayesian inferring model are not the point of this article. So the details of the algorithms of the Bayesian inferring model can be found in the former work (Liu, 2010).

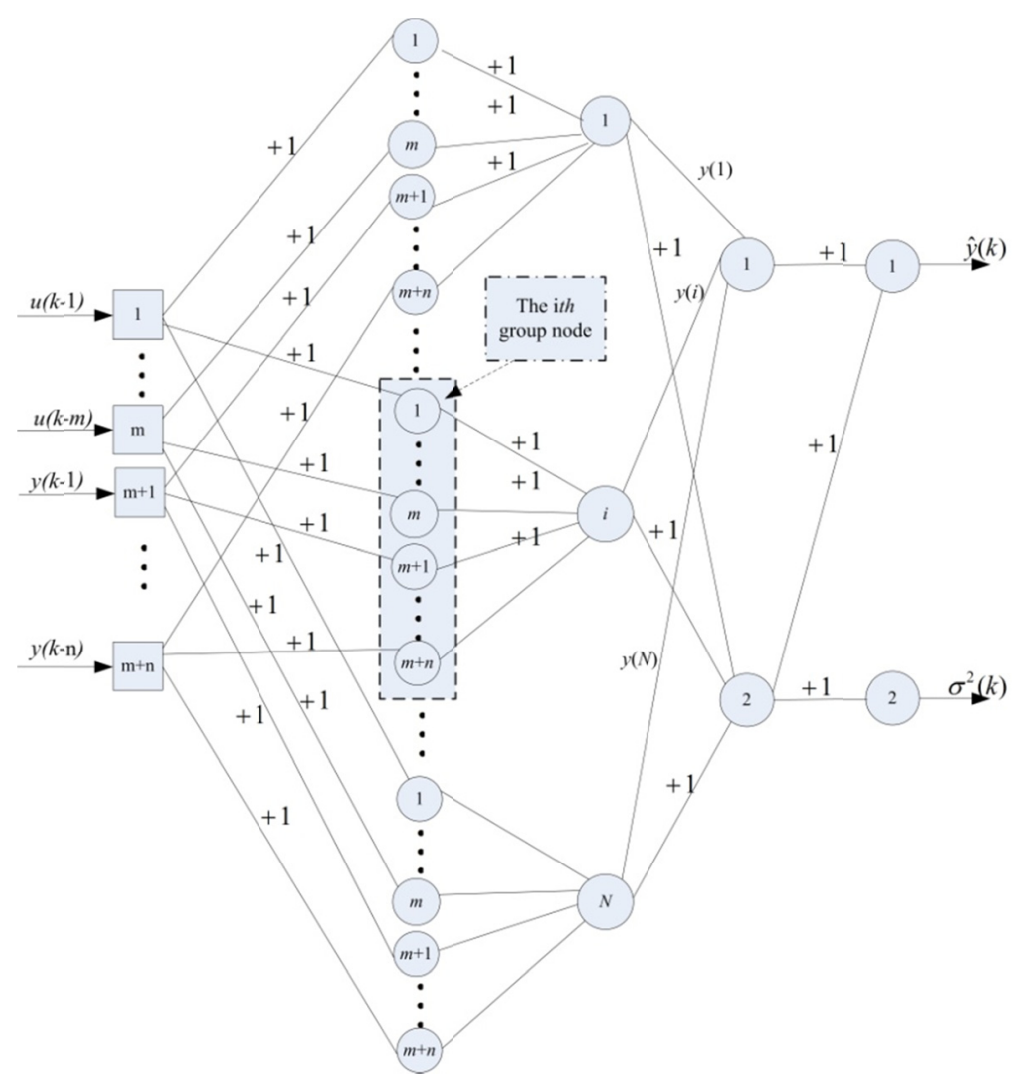

Figure 2. Illustration of Bayesian inferring model structure

\subsection{Receding Horizon of Control Variable}

The most classical characteristic of model predictive control is that the control variable $u(k)$ should be obtained through the on-line receding horizon method. The objective function is selected as the following formula.

$$
J(k)=\sum_{j=1}^{P}\left[y_{r}(k+j)-y_{p}(k+d)\right]^{2}+\sum_{i=1}^{M}[\Delta U(k+i-1)]^{2}
$$

Where $P$ and $M$ denote the predictive and control time horizons; $\Delta U(k)=u(k)-u(k-1) ; y_{r}(k+i)$ is the $i^{\text {th }}$ desired system output at the time moment of $k$ and determined by the following equation.

$$
y_{r}(k+j)=c^{j} y(k)+\left(1-c^{i}\right) r(k)
$$

where $r$ is the reference input signal; $c$ is called flexible coefficient designed according to the controlled object.

From the construction of the Bayesian model predictive control, $J(k)$ is the nonlinear function about the $u(k+i), i=1, \ldots, M$. Therefore, the optimization of the control variables is converted to the minimization of the 
objective function $J(k)$. Namely, in the feasible control field of $\left[u_{\min }, u_{\max }\right]$, the optimization problem is to obtain a feasible solution $U^{*}=\left[u^{*}(k), \ldots, u^{*}(k+M)\right]^{T}$ to make the $J(k)$ minimum. In the implementation of control, only the $u^{*}(k)$ is used to act on the controlled object at the $k$ time moment. Then at the time moment $k+1$, the optimization progress is repeated as the moment $k$. And in this way, the whole dynamic process of the controlled object is optimized.

The above receding horizon of $u^{*}(k)$ belongs to the nonlinear programming problem with constraint conditions. In this work, we choose the steepest gradient method as the receding horizon algorithm. Taking the comprehensive consideration of the training of the Bayesian inferring predictive model and the working flow of the BMPC, we give the implementation procedures of the BMPC method.

(1) Obtain the sample data of the controlled object. Determine the input and output vector of the Bayesian inferring model. Utilize the PSO algorithm (Vahid, 2013; Cabrerizo, 2013) to train the parameters of the threshold matrix $D$.

(2) Determine the width of the sliding window $N$ and validate the on-line predictive ability of the obtained Bayesian inferring model.

(3) Based on the trained Bayesian inferring model, the implementation of the BMPC is set as follows.

a) Obtain the current output $y(k)$ and then compute the Bayesian inferring model output $\hat{y}(k)$. The error $e(k)$ is computed as $y(k)-\hat{y}(k)$.

b) Utilize the Bayesian inferring model to computer the $\hat{y}(k+i), i=1, \ldots, P$; according to the formula 1 , compute the corrected predictive output $y_{p}(k+i)$; at the same time, compute the desired system output $y_{r}(k+i)$ according to the formula 7 .

c) Use the steepest gradient method to solve the minimization of the objective function $J(k)$ and the optimized control variable $u^{*}(k)$ is obtained.

d) Take the $u^{*}(k)$ to implement on the controlled object. Then go to the step a) to continue the next optimization procedure at the time moment $k+1$.

\section{Convergence Analysis of Bayesian Model Predictive Controller}

The objective function $J(k)$ is described as the vector form.

$$
J(k)=\left[Y_{r}-Y_{p}\right]^{T}\left[Y_{r}-Y_{p}\right]+\Delta U^{T} \Delta U
$$

where, $Y_{r}=\left[y_{r}\left(k+1, \ldots, y_{r}(k+P)\right)\right]^{T}, \quad Y_{P}=\left[y_{p}\left(k+1, \ldots, y_{P}(k+P)\right)\right]^{T}, \Delta U=[\Delta U(k), \ldots, \Delta U(k+M-1)]$.

Let $E=Y_{r}-Y_{P}, J(k)$ is the function about $U$. According to the steepest gradient method (Zhang, 2010), the optimal control law is listed as follows.

$$
U(k)^{T}=U(k-1)^{T}-\frac{\lambda}{1+\lambda} E(k+1)^{T} \frac{\partial E(k+1)}{\partial U(k)}
$$

where, $\lambda$ denotes the optimized step length, $\lambda>0$.

According to the above formula

$$
E(k+1)^{T} \frac{\partial E(k+1)}{\partial U(k)}=-\Delta U(k)^{T} \frac{\lambda}{1+\lambda}
$$

Obtain the one first order derivative of the $J(k)$

$$
\frac{\partial J(k)}{\partial k}=2 E(k+1)^{T} \frac{\partial E(k+1)}{\partial U(k)} \frac{\partial U(k)}{\partial k}+2 \Delta U(k)^{T} \frac{\partial U(k)}{\partial k}
$$

The approximation of the first order derivative is set as

$$
\frac{\Delta J(k)}{\Delta k}=2 E(k+1)^{T} \frac{\partial E(k+1)}{\partial U(k)} \frac{\Delta U(k)}{\Delta k}+2 \Delta U(k)^{T} \frac{\Delta U(k)}{\Delta k}
$$

Take the formula 10 into the above equation and the obtain the following formula

begin $\{$ equation 


$$
\Delta J(k)=\left(2-\frac{2+2 \lambda}{\lambda}\right)\left[\Delta U(k)^{T} \Delta U(k)\right]=\frac{-2}{\lambda}\|\Delta U(k)\|^{2}
$$

In order to ensure the convergence of the optimization algorithm, the objective function $J(k)$ should be non-increasing function. From the above analysis, $\Delta J(k)$ is satisfied. The convergence of the BMPC is proven.

\section{Simulation Case Studies}

To investigate the performance of the Bayesian model predictive controller, the experiments are conducted on the closed loop control of two kinds of controlled object, namely the nonminimum phase and nonlinear objects. In the experiments, the control effectiveness, anti-disturbance ability and robust performance are observed.

\subsection{Description of the Controlled Objects}

\subsubsection{Nonminimum Phase Object}

In the field of electrical systems, there are many nonminimum controlled objects such as the thermal power generation systems, the hydraulic generator system, etc. Because of the large phase lag, the nonminimum systems are hard to control with the slow response of system output.

The nonminimum system with the following transform function is chosen as the first experimental object.

$$
Y(s)=\frac{1-3.5 s}{(1+1.75 s)(1+8.5 s)} U(s)
$$

\subsubsection{Nonlinear Object}

There are many kinds of nonlinear characteristics in industrial process. The following difference equation describes the second experimental controlled object as a nonlinear object example.

$$
\begin{gathered}
y(k)=\left(0.8-0.5 e^{-y^{2}(k-1)}\right) y(k-1) \\
-\left(0.3+0.9 e^{-y^{2}(k-1)} y(k-2)\right)+u(k-1)+0.2 u(k-2)+0.1 u(k-1) u(k-2)+e(k)
\end{gathered}
$$

where, $e(k)$ denotes the white gaussian noise with zero mean and variance value 0.04 .

\subsection{Simulation Procedures}

\subsubsection{Training and Testing of Bayesian Predictive Model}

First, it is needed to obtain the sample data for the training and testing of the Bayesian predictive models. In the experiments, the control variable $u(k)$ adopts the one mean and the variance value 0.2 and the 1000 groups of output sample data $y(k)$ are shown in figure 3. The former 500 groups of data are used for the training of the Bayesian predictive models and the next 500 groups of data are for the validation of the predictive effectiveness of the trained models.
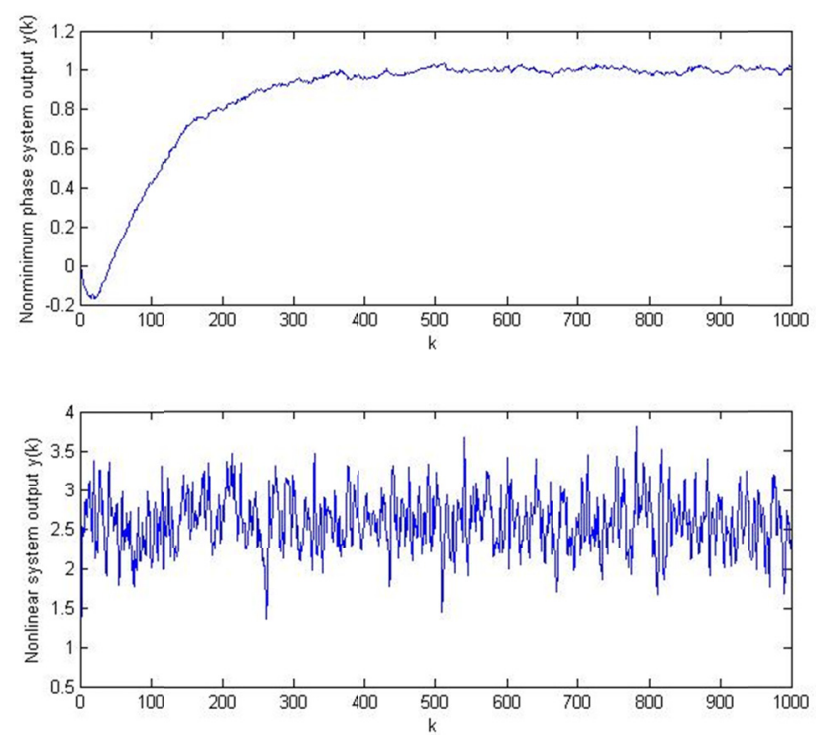

Figure 3. Sample data for system output 
In the off-line training of the Bayesian predictive models, the parameters in the particle swarm optimization algorithms are selected as: the swarm number $S=20, c_{1}=0.5, c_{2}=0.5$. The structure of the bayesian predictive model for the nonminimum phase object is set as the input vector $X=[u(k), u(k-1), y(k-1)]^{T}$ and the output $y(k)$. According to the choice of the structure, there is 3 parameters needed to be optimized in the threshold $D$. The structure of the bayesian predictive model for the nonlinear controlled object is selected as the input vector $X=[u(k), u(k-1), y(k-1), y(k-2)]^{T}$ and four parameters in the threshold matrix $D$ are to be optimized.

After the training of the particle swarm optimization, the threshold matrix $D$ of the Bayesian predictive model for the nonminimum phase object is then obtained $D=\operatorname{diag}[26.5656,23.4290,79.4851]$. And the threshold matrix $D$ for the nonlinear object is set as $D=\operatorname{diag}[189.8301,53.6387,124.6788,38.6437]$. The width of the sliding window $N$ is selected as 3 . According to the next 500 groups of testing sample, the on-line predictive output curves are shown in figure 4.
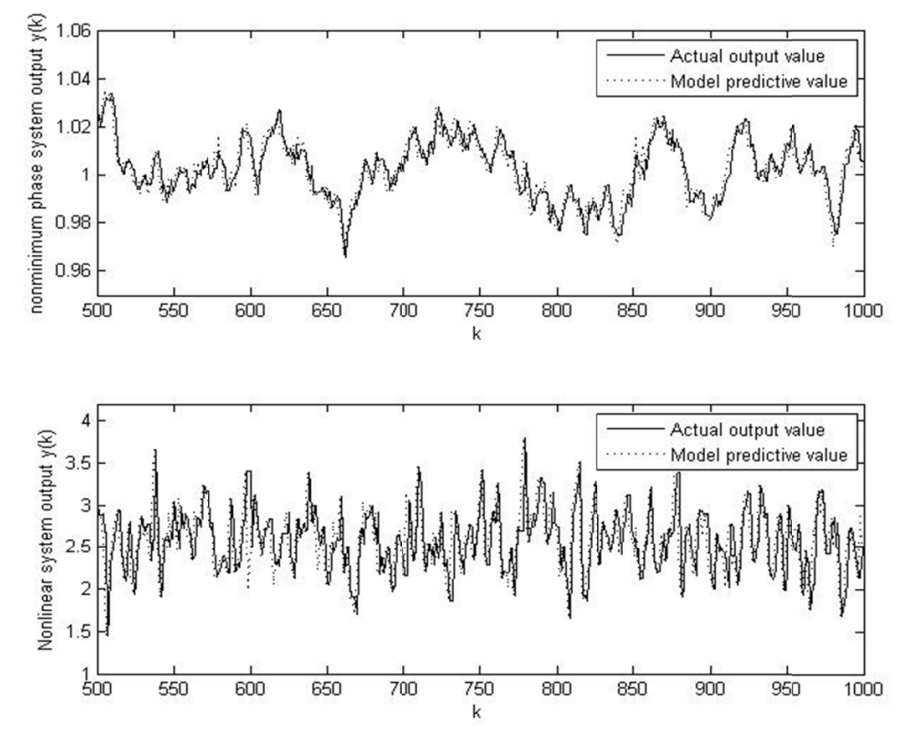

Figure 4. On-line prediction of Bayesian inferring model

\subsubsection{Bayesian Model Predictive Control}

In the second procedure, the above obtained Bayesian predictive models are used to construct the closed model predictive control of the nonminimum object and the nonlinear object. In the two closed loop controls, the reference signals are all constant value.

In the control of the nonminimum system, the relevant parameters are set as the predictive horizon $P=6, M=6$ and the flexible coefficient $c=0.6$. In order to observe the anti-disturbance ability and robust performance of the Bayesian model predictive control system, the random disturbances with amplitude 0.1 are added at the moments of the $k=60$ and the $k=120$. Then the control curve of the Bayesian model predictive controller is shown in figure 5 . 


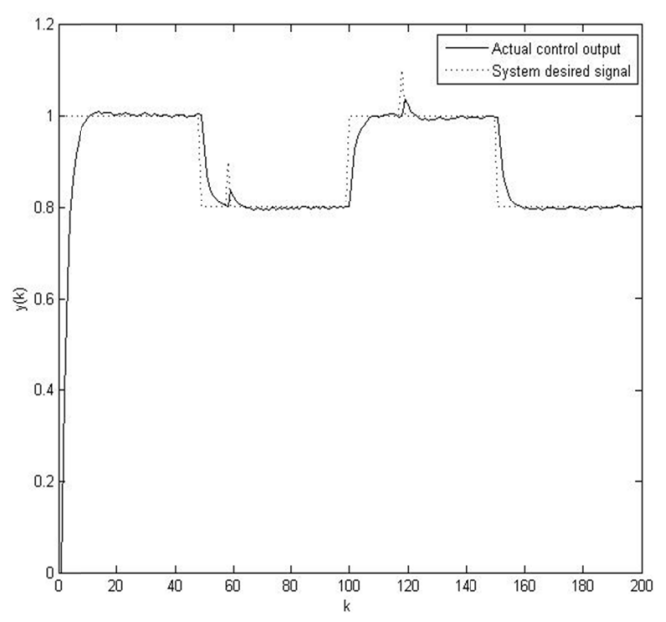

Figure 5. Control result of nonminimum phase system based on BMPC

In the control of the nonlinear object, the relevant parameters are set as the predictive horizon $P=6, M=6$ and the flexible coefficient $c=0.4$. The random disturbances with amplitude 0.05 are added at the moments of the $k=60$ and the $k=120$. Then the control curve of the Bayesian model predictive controller is shown in figure 6.

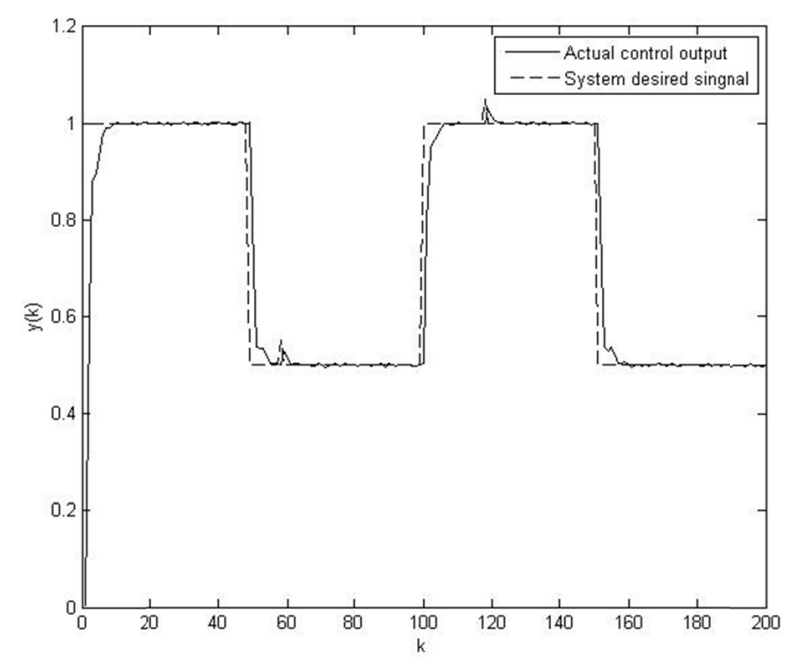

Figure 6. Control result of nonlinear system based on BMPC

\section{Results Analysis}

From the above experiments, it can be seen that the training of the Bayesian predictive is very simple and the parameters needed to be optimized are merely the ones in the threshold matrix $D$. When the Bayesian predictive model is used for the on-line prediction, it can capture the nonlinear characteristics quickly and have high prediction accuracy. The reason for its characteristic is that the Bayesian predictive model can update its structure using on-line sample data.

In the closed loop of Bayesian model predictive control, the steepest gradient method ensures the convergence ability of receding horizon algorithm. Therefore, the control variable $U^{*}$ at every step can obtain the optimized solution. From the analysis of the experiments, the Bayesian model predictive control obtains desired control effectiveness. When the closed control system have strong disturbance, the Bayesian model predictive control system also achieves stability because the Bayesian predictive model can capture the nonlinear characteristics quickly. 


\section{Conclusion}

In this note, the Bayesian model predictive control system is designed based on the Bayesian inferring model. The particle swarm optimization algorithm is used to the off-line training of the Bayesian inferring model. In the on-line implementation of the Bayesian inferring model, the sliding window method is utilized to achieve the structure updating so as to capture the nonlinear characteristics of system quickly. The receding horizon optimization in the design of the Bayesian model predictive controller adopts the steepest gradient method, which ensures the convergence ability of the proposed model predictive control algorithm. And the experiments implemented on the nonminimum phase and nonlinear systems show that the Bayesian model predictive control system owns good control effect, anti-disturbance ability and robust performance.

\section{Acknowledgements}

The authors would like to thank the support of the National Natural Science Foundation of China (No.61304227, No.61273114) and the Jiangsu Government Scholarship for Overseas Studies (2013).

\section{References}

Ali, M., Zoltan, K. N., Adrie, E. H., Herman, J. K., \& Paul M. V. den Hof. (2012). Nonlinear model-based control of a semi-industrial batch crystallizer using a population balance modeling framework. Control $\begin{array}{lllll}\text { Systems Technology, IEEE Transactions } & \text { on, }\end{array}$ http://dx.doi.org/10.1109/TCST.2011.2160945

Anders Willersrud et al. (2013). Short-term production optimization of offshore oil and gas production using nonlinear model predictive control. Journal of Process Control, 23(2), 215-223. http://dx.doi.org/10.1016/j.jprocont.2012.08.005

Armando, D., Assandri et al. (2013). Nonlinear parametric predictive temperature control of a distillation column. Control Engineering Practice, 21(12), 1795-1806. http://dx.doi.org/10.1016/j.conengprac.2013.09.004

Aswani et al. (2013). Provably safe and robust learning-based model predictive control. Automatica, 49(5), 1216-1226. http://dx.doi.org/10.1016/j.automatica.2013.02.003

Cabrerizo et al. (2013). A method based on PSO and granular computing of linguistic information to solve group decision making problems defined in heterogeneous contexts. European Journal of Operational Research, 230(3), 624-633. http://dx.doi.org/10.1016/j.ejor.2013.04.046

Christofides et al. (2013). Distributed model predictive control: A tutorial review and future research directions. Computers \& Chemical Engineering, 51, 21-41. http://dx.doi.org/10.1016/j.compchemeng.2012.05.011

Giselsson et al. (2013). Accelerated gradient methods and dual decomposition in distributed model predictive control. Automatica, 49(3), 829-833. http://dx.doi.org/10.1016/j.automatica.2013.01.009

Karim Salahshoor et al. (2013). Stabilization of gas-lift oil wells by a nonlinear model predictive control scheme based on adaptive neural network models. Engineering Applications of Artificial Intelligence, 26(8), 1902-1910. http://dx.doi.org/10.1016/j.engappai.2013.03.007

Lincoln, F. L., Moro et al. (2013). A mixed-integer model predictive control formulation for linear system. Computer and Chemical Engineering, 55, 1-18. http://dx.doi.org/10.1016/j.compchemeng.2013.04.011

Liu Yijian et al. (2010). Modeling of hydraulic turbine system based on a bayesian-gaussian neural network driven by sliding window data. Journal of Zhejiang University. Science. C, 11(1), 56-62. http://dx.doi.org/10.1631/jzus.C0910176

Mesbah, A., Landlust, J., AEM H., HJM K., Jansens, P. J., \& PMJ Van den Hof. (2010). A model-based control framework for industrial batch crystallization processes. Chemical Engineering Research and Design, 88(9), 1223-1233. http://dx.doi.org/10.1016/j.cherd.2009.09.010

Hazil, O. et al. (2014). Fuzzy model predictive control of dc-dc converters. In AETA 2013: Recent Advances in Electrical Engineering and Related Sciences, 423-432.

Rodriguez et al. (2013). State of the art of finite control set model predictive control in power electronics. Industrial Informatics, IEEE Transactions on, 9(2), 1003-1016. http://dx.doi.org/10.1109/TII.2012.2221469

Tung Le et al. (2013). Linear-quadratic model predictive control for urban traffic networks. Transportation Research Part C: Emerging Technologies, 36, 498-512. http://dx.doi.org/10.1016/j.trc.2013.06.021

Vahid, F., \& Gholam Ali, M. (2013). An improvement in RBF learning algorithm based on PSO for real time applications. Neurocomputing, 111, 169-176. http://dx.doi.org/10.1016/j.neucom.2012.12.024 
Xiangjie, L., \& Xiaobing, K. (2013). Nonlinear fuzzy model predictive iterative learning control for drum-type

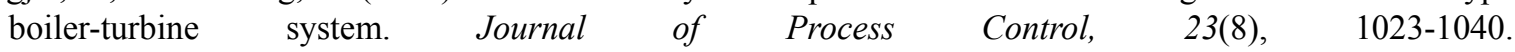
http://dx.doi.org/10.1016/j.jprocont.2013.06.004

Zhang, L. (2010). Optimization Method. Beijing: Science Press.

\section{Copyrights}

Copyright for this article is retained by the author(s), with first publication rights granted to the journal.

This is an open-access article distributed under the terms and conditions of the Creative Commons Attribution license (http://creativecommons.org/licenses/by/3.0/). 